Supporting Information for

Marine and Anthropogenic Bromopyrroles Alter Cellular $\mathrm{Ca}^{2+}$ Dynamics of Murine Cortical Neuronal Networks by Targeting Ryanodine Receptor and Sarco/Endoplasmic Reticulum $\mathrm{Ca}^{2+-}$ ATPase

Jing Zheng ${ }^{1}$, Shane Antrobus ${ }^{1}$, Wei Feng ${ }^{1}$, Trevor N. Purdy ${ }^{2,3,4}$, Bradley S. Moore ${ }^{2,}$ ${ }^{3,4}$ and Isaac N. Pessah ${ }^{1}$

${ }^{1}$ Department of Molecular Biosciences, School of Veterinary Medicine, University of California, Davis, CA 95616, USA

${ }^{2}$ Center for Marine Biotechnology and Biomedicine, University of California, San Diego, CA 92037, USA

${ }^{3}$ Scripps Institution of Oceanography, University of California, San Diego, CA 92037, USA

${ }^{4}$ Skaggs School of Pharmacy and Pharmaceutical Sciences, University of California, San Diego, CA 92037, USA

Correspondence to:

Isaac N. Pessah

Department of Molecular Biosciences

School of Veterinary Medicine

1089 Veterinary Medicine Drive

UC Davis 95616

$530-752-6696$

inpessah@ucdavis.edu 
Number of pages: 17

Number of tables: 1

Number of figures: 3 


\section{Table of Content:}

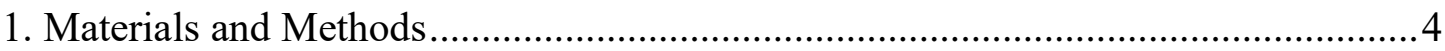

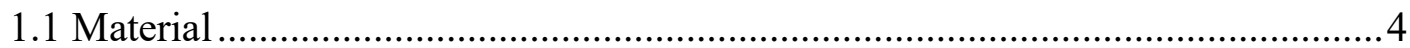

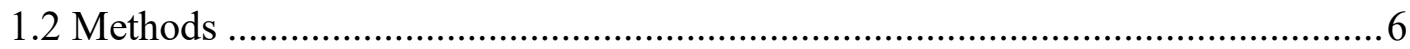

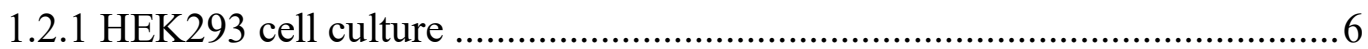

1.2.2 Validation of RYR1 and SERCA expression using WB and ICC ................6

1.2.3 Measurement of intracellular $\mathrm{Ca}^{2+}$ in HEK293 cell lines using FLIPR........ 7

1.2.4 Primary cortical neuronal/glial coculture (NGC) .................................. 8

1.2.5 Measurements of intracellular $\mathrm{Ca}^{2+}$ dynamics of NGC ..............................9

1.2.6 NGC electrical spike activity (ESA) measurement .................................. 10

1.2.7 Measurement of neuronal viability ........................................................ 11

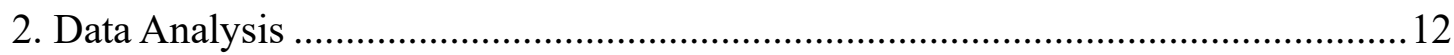

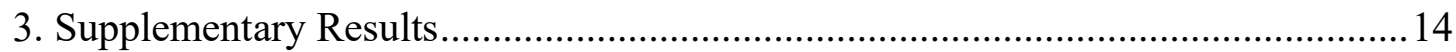

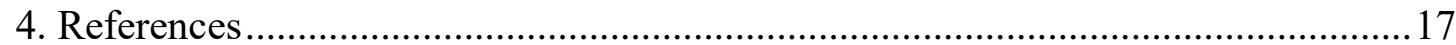

\section{Tables:}

Table S1 List of chemicals and reagents used in this study .4

\section{Figures:}

Figure S1 Representative western blot probed with RYR monoclonal antibody 34C and GAPDH polyclonal antibody showing molecular mass markers (in kDa). 14

Figure S2 Initial rates of $\mathrm{Ca}^{2+}$ transients induced by TBP in null and RYR1-expressing HEK293 cell lines.

Figure S3 TBP at concentrations that fully inhibit SCO activity does not alter electrical spike activity of neuronal networks. 


\section{Materials and Methods}

\subsection{Material}

Table S1 List of chemicals and reagents used in this study.

\begin{tabular}{|c|c|c|}
\hline Name of chemicals and reagents & Source & Catalog number \\
\hline AlexaFluor-488 conjugated & Thermo Fisher & A21121 \\
\hline goat anti-mouse & (San Diego, CA) & \\
\hline AlexaFluor-555 conjugated & Thermo Fisher & A21437 \\
\hline goat anti-chicken & (San Diego, CA) & \\
\hline Cytosine $\beta$-D-arabinofuraboside, & Life Technology (Grand Island, NY) & C1768-100MG \\
\hline Chicken anti-MAP-2B & Thermo Fisher (San Diego, CA) & APPA6751 \\
\hline Caffeine & Sigma-Aldrich (St. Louis, MO) & $\mathrm{C} 0750$ \\
\hline DNase I & Life Technology (Grand Island, NY) & D5025-150KU \\
\hline Dulbecco's Modified Eagle Medium & Life Technology (Grand Island, NY) & 11965-092 \\
\hline Dimethyl sulfoxide & VWR Life Science (Radnor, PA) & BDH1115- \\
\hline $\begin{array}{l}\text { Ethyl 4-bromopyrrole-2-carboxylate } \\
\qquad \text { (5) }\end{array}$ & Sigma-Aldrich (St. Louis, MO) & $\begin{array}{l}1 \mathrm{LP} ;>99.9 \% \\
729787 ; 97 \%\end{array}$ \\
\hline Fluo-4/AM & Life Technology (Grand Island, NY) & F14202 \\
\hline Fetal bovine serum & Atlanta Biologicals (Norcross, GA) & A93E-500ml \\
\hline Gluta-Max & Life Technology (Grand Island, NY) & 35050061 \\
\hline G-418 & Life Technology (Grand Island, NY) & 10131035 \\
\hline GS21 supplement & $\begin{array}{c}\text { MTI-Global Stem (Gaithersburg, } \\
\text { MD) }\end{array}$ & GSM-3100 \\
\hline $10 \%$ Goat serum & Life Technology (Grand Island, NY) & $50062 Z$ \\
\hline $\begin{array}{c}\text { IRDye } 800 \mathrm{CW} \text { conjugated goat anti- } \\
\text { mouse IgG1 }\end{array}$ & Li-Cor Biosciences (Lincoln, NE) & $926-32350$ \\
\hline L-glutamine & Life Technology (Grand Island, NY) & 25030081 \\
\hline
\end{tabular}


Mouse anti-GFAP

Mouse anti-GAPDH

Mouse anti-RYR1 primary antibody $34 \mathrm{C}$

Mouse anti-SERCA

MTT (3-(4,5-Dimethylthiazol-2-yl)-

2,5-Diphenyltetrazolium Bromide)

NucBlue Fixed Cell Ready Probes

Reagent (DAPI)

Neurobasal medium

Penicillin-Streptomycin

Poly-L-lysine

Pyrrole (3)

Ryanodine

Soybean trypsin inhibitor

Tetrabromopyrrole (1)

2,3,5-tribromopyrrole (2)

2,3,4-tribromopyrrole (4)

Trypsin
Cell Signaling (Danvers, MA)

$3670 \mathrm{~S}$

Millipore, Billerica, MA

Mab 374

Developmental Studies Hybridoma

DSHB Cat\# 34c

Bank (Iowa City, IA)

Developmental Studies Hybridoma

$\mathrm{CaS} / \mathrm{C} 1$ or $3 \mathrm{H} 2$

Bank (Iowa City, Iowa)

Thermo Fisher (San Diego, CA)

M6494

Life Technology (Grand Island, NY)

R37606

Life Technology (Grand Island, NY)

12348017

Sigma-Aldrich (St. Louis, MO)

P4333

Peptides International Inc.

P2636-100MG

(Louisville, KE)

Sigma-Aldrich (St. Louis, MO)

131709; >98\%

Abcam/Ascent Scientific

Ab120083;

(Pleasanton, CA)

$\geq 98.3 \%$

Atlanta Biologicals (Norcross, GA)

T9128-1G

Scripps Institution of Oceanography

54705-14-5

(San Diego, CA)

Scripps Institution of Oceanography

77124-07-3

(San Diego, CA)

Scripps Institution of Oceanography

69624-12-0

(San Diego, CA)

Atlanta Biologicals (Norcross, GA)
I56201 


\subsection{Methods}

\subsubsection{HEK293 cell culture}

HEK 293 cell line (CRL-1573) obtained from ATCC (Manassas, VA) was transfected with RyR1 as previously reported ${ }^{1}$. Cryopreserved RYR-null HEK293 and RYR1-expressing HEK293 cell lines were quickly thawed, diluted, plated and expanded on $75 \mathrm{~cm}^{2}$ flasks in supplemented DMEM. After the second passage, cells were plated onto poly-L-lysine pre-coated 96-well imaging plates at 30,000 cells/well, and then maintained at $37{ }^{\circ} \mathrm{C}$ with $5 \% \mathrm{CO}_{2}$ and $95 \%$ humidity for $24 \mathrm{~h}$. Presence or lack of RYR1 expression was verified using three approaches; (1) western blotting (WB), (2) fluorescent immunocytochemistry (ICC) and (3) testing for the presence or absence of functional responses to caffeine $(100 \mu \mathrm{M})$ in Fluo-4 loaded cells.

\subsubsection{Validation of RYR1 and SERCA expression using WB and ICC}

$W B$ : The conditions used during electrophoresis for the western blot are described previously $^{2}$. RyR1 was detected with antibody 34C (1:1000) purchased from the Developmental Studies Hybridoma Bank, University of Iowa, Iowa), and SERCA was detected using anti-SERCA (1:2000) from Developmental Studies Hybridoma Bank (DSHB); University of Iowa, Iowa. Housekeeping protein GAPDH was detected by polyclonal mouse anti-GAPDH (1:2500) (Millipore, Billerica, MA). PVDF membranes were incubated with infrared fluorescent IRDye $800 \mathrm{CW}$ conjugated secondary goat anti-mouse IgG1antibodies (1:1000) (Li-Cor Biosciences, Lincoln, NE). The membranes were scanned with a LI-COR Odyssey infrared imager (LI-COR Biosciences, Linclon, NE) and band intensity was analyzed using Odyssey version 3.0 software. Blots were repeated on $>3$ separate occasions to verify expression differences between WT and RYR1-expressing lines. 
ICC: HEK293 cells grown on coverslips were fixed with 4\% paraformaldehyde in $2 \%$ sucrose. The cells were permeabilized with triton X-100 (0.25\% in PBS) and incubated in goat serum (1h). The cells were then incubated in anti-RyR antibody 34C (1:25) (Developmental Studies Hybridoma Bank, University of Iowa, Iowa) overnight at $4^{\circ} \mathrm{C}$. Staining was then carried out with AlexaFluor-488 conjugated anti-mouse secondary antibody (1:1000) (Invitrogen, CA), followed by nuclear staining using 2 drops per ml of NucBlue ${ }^{\mathrm{TM}}$ Fixed Cell ReadyProbes ${ }^{\mathrm{TM}}$ Reagent (Molecular Probes, Life Technology). The coverslips were fixed on glass slides with syber gold (Invitrogen, CA) and the images were collected with an Olympus IX71 epifluorescence microscope equipped with a CCD camera using a 40X objective.

\subsubsection{Measurement of intracellular $\mathrm{Ca}^{2+}$ in HEK293 cell lines using FLIPR}

Once the HEK293 lines were validated for RYR1 expression, Fluo-4 loaded cells were placed in a fluorescent imaging plate reader (FLIPR ${ }^{\text {Tetra }}$, Molecular Devices) to measure temporal changes in intercellular $\mathrm{Ca}^{2+}$ simultaneously across multiple wells. After a 5 min stabilization period, baseline fluorescence was recorded for 2 min and subsequently exposed to caffeine $(100 \mu \mathrm{M})$ with the additions of vehicle $(0.1 \%$ DMSO) or TBP $(0.1-2.0 \mu \mathrm{M})$. A programmable multichannel robotic pipettor was used to transfer test solutions from a source plate containing the vehicle control or $7 \mathrm{x}$ concentrated stock to the target plate containing the cells. Changes in intracellular concentrations recorded for $120 \mathrm{~s}$ at $0.5 \mathrm{~Hz}$ in the presence of a replete (physiological) extracellular $\mathrm{Ca}^{2+}$ concentration $(2.3 \mathrm{mM})$ and repeated on a set of separate cultures with extracellular free $\mathrm{Ca}^{2+}$ buffered with EGTA to a concentration of $0.3 \mathrm{mM}$ to directly assess release from SR/ER stores. Wells with no-addition and vehicle controls were present on each experimental plate to serve as experimental controls. 
Data analysis of responses of HEK293 cell lines- Data obtained from HEK293 cells were analyzed from at least four technical replicates in each well and were repeated on at least three separate culture days to assess biological variability. Data of initial rate and $\mathrm{Ca}^{2+}$ transient peak amplitude $\left(\triangle \mathrm{F} / \mathrm{F}_{0}\right)$ expressed as mean \pm standard error of mean (SEM) were obtained from three independent cultures, with four replicates each. The curves of initial rate and amplitude as function of test concentration were fitted by non-linear regression using GraphPad Prism software (Version 7.03, GraphPad Software Inc., San Diego, CA).

\subsubsection{Primary cortical neuronal/glial coculture (NGC)}

Animal related experiments were conducted in accordance with the guidelines of Animal Use and Care of the National Institutes of Health and approved by the University of California Davis Animal Use and Care Committee. The dissection procedures of cortical neurons from postnatal day 0-1 (male and female brains were pooled) C57BL/6J mice pups were as previous described ${ }^{3}$. Briefly, the dissociated cells were suspended in Neurobasal complete (NBC) medium (Neurobasal medium supplemented with GS21 at 2\% v/v, Gluta-Max at 1\% v/v, $10 \mathrm{mM} \mathrm{HEPES)} \mathrm{with} \mathrm{5 \%}$ FBS. Then the suspended cells were plated onto poly-L-lysine $(0.5 \mathrm{mg} / \mathrm{mL}$ in borate buffer) pre-coated 96-well imaging plate (BD, Franklin Lakes, NJ) and maintained at $37{ }^{\circ} \mathrm{C}$ with $5 \% \quad \mathrm{CO}_{2}$ and $95 \%$ humidity. For neuronal viability, intracellular synchronized $\mathrm{Ca}^{2+}$ oscillations (SCOs) and electrophysiological spike activity (ESA) measurements, the plating densities were 45,000, 75,000 and 130,000 cells/well, respectively. A final concentration of $5 \mu \mathrm{M}$ of cytosine $\beta$-D-arabinofuraboside dissolved in FBS-free NBC was added to each well at 24-30 $\mathrm{h}$ after plating. Half of the medium was changed with freshly made FBS-free NBC at one day before measurements were 
performed.

ICC characterization of the presence and proportion of astrocyte in neuronal model- The 7 days in vitro (DIV) neurons with Ara-C treatment that were grown in the black wall clear-bottom 96-well plate were fixed with 4\% paraformaldehyde for $10 \mathrm{~min}$ at room temperature (RT), followed by PBS rinse for 4 times with 5 min each time at RT. The cells were then permeabilized with $0.25 \%$ Triton X-100 in PBS for $5 \mathrm{~min}$. Following being blocked with $10 \%$ goat serum for $1 \mathrm{hr}$ at RT, the cells were incubated with chicken anti-MAP-2B (1:2000) and mouse anti-GFAP (1:2000) primary antibodies in PBS containing $10 \%$ goat serum for overnight at $4{ }^{\circ} \mathrm{C}$. After 4 times ( 5 min each) of rinse with PBS, the cells were then incubated with AlexaFluor-488 conjugated goat anti-mouse (1:500) and AlexaFluor-555 conjugated goat anti-chicken (1:500) secondary antibodies for $1 \mathrm{hr}$ at RT. After adding 2 drops per ml of NucBlueVR Fixed Cell ReadyProbesVR Reagent (Molecular Probes, Life Technology) for nuclei staining for $5 \mathrm{~min}$ and performing 4 times of rinse with PBS, the cells were imaged with an ImageXpress ${ }^{\circledR}$ High Content Imaging System (Molecular Devices) using a $20 \times$ objective with fluorescein isothiocyanate filter.

Cell count- The number of MAP-2B positive and GFAP positive cells were manually measured using Image J software (version 1.49s; U.S. National Institutes of Health) from images taken from three random positions each well. All data were express as mean \pm SEM from two independent cultures, each data point represent an average of three positions in a single well.

\subsubsection{Measurements of intracellular $\mathrm{Ca}^{2+}$ dynamics of NGC}

At $7 \mathrm{DIV}$, neurons were incubated for $1 \mathrm{~h}$ with $4 \mu \mathrm{M}$ Fluo- 4 plus $0.5 \mathrm{mg} / \mathrm{mL}$ BSA in Locke's buffer consisting of (in mM) 8.6 HEPES, 5.6 KCl, $154 \mathrm{NaCl}$, 5.6 glucose, 
1.0 $\mathrm{MgCl}_{2}, 2.3 \mathrm{CaCl}_{2}$, and 0.1 glycine, $\mathrm{pH}$ 7.4. As previously described ${ }^{4}$, neurons were then gently rinsed with pre-warmed Locke's buffer for 4 times, with a final volume of $175 \mu \mathrm{L} /$ well. After being loaded onto the imaging chamber of the FLIPR for 5 min for equilibration, SCOs were recorded for $120 \mathrm{~s}$ followed by the addition of vehicle or concentrations of test compounds indicated in the figures (delivered in $25 \mu \mathrm{L}$ in Locke's buffer at $8 \mathrm{x}$ stock) using a programmable 96-channel robotic pipetting system. Fluorescent signals (F) were recorded for additional 1,380 s at a sampling rate of $1 \mathrm{~Hz}$.

SCO analyses - For SCO analysis, the data was presented as $\triangle \mathrm{F} / \mathrm{F}_{0}$, where $\mathrm{F}_{0}$ is the minimal arbitrary fluorescence unit before addition, and $\triangle F$ is the result of fluorescent value subtracted by $F_{0}$. An event with amplitude of $\triangle F / F_{0}>0.15$ was considered to be an SCO. Frequency and amplitude data were generated by Origin software (version 9.0; Origin Lab Corporation). As the initial several minutes produce artificial effects on SCO pattern, thus data collected from 5-23 min after addition were used to evaluate the effect of test compounds. For intracellular $\mathrm{Ca}^{2+}$ elevation, the area under calcium release curves between 120-1200 s after recording were summarized and used for assessment of BrPyr-trigged responses. All the data expressed as mean $\pm \mathrm{SEM}$ were obtained from three independent cultures, with six replicates each. Non-linear regression or one-way ANOVA followed by Bonferroni's multiple comparisons test with Prism (Version 7.03, GraphPad Software Inc., San Diego, CA) was respectively used to fit the curves or to compare the difference of mean between vehicle control (Veh) and test compounds treated groups.

\subsubsection{NGC electrical spike activity (ESA) measurement}

Neuronal network ESA was recorded using neurons/glia cultured on 12-well microelectrode array (MEA) plates (Axion BioSystems, Atlanta, GA) as previously 
described $^{4}$. Briefly, ESA were recorded from 7 DIV cortical neurons using AxIS software (Version 2.4, Axion BioSystems). After 5 min equilibration, ESA activity was acquired for $10 \mathrm{~min}$ (baseline) followed by addition of vehicle or the indicated concentrations of compound $\mathbf{1}$ (TBP) to record for an additional 10 min, at a sampling frequency of $12.5 \mathrm{kHz}$ filtered at cutoff of $200 \mathrm{~Hz}-3 \mathrm{kHz}$ with a Butterworth band-pass filter. AxIS software was used to detect spontaneous events with a threshold of $\geq 8$ times of the noise amplitude, and the Neural Offline 'Spontaneous' configuration was run to export parameters including number of active electrodes, spikes, bursts, and network bursts. Representative raster plots were with NeuroExplorer software (version 4.0, NEX Technologies, Littleton, MA). For all parameters analyzed (spikes, bursts and network bursts) each MEA well was normalized to its corresponding baseline prior to being normalized to the corresponding average value of the Veh wells on the same plate and expressed as \% Veh. All data in the bar graph presented as mean \pm SEM were collected from four independent culture, with four replicates each. One-way ANOVA followed by post hoc Bonferroni multiple comparison were used to determine the statistical significance of mean values of parameters between Veh and TBP-treated groups with Prism (Version 7.03, GraphPad Software Inc., San Diego, CA).

\subsubsection{Measurement of neuronal viability}

Cortical NGC were exposed to vehicle or BrPyr at 7 DIV for $24 \mathrm{~h}$, and the MTT assay was used to evaluate the neuronal toxicity as previously published ${ }^{5}$. Briefly, $24 \mathrm{~h}$ after vehicle or BrPyr exposures, the medium was aspirated and a volume of $100 \mu \mathrm{L}$ medium containing $0.5 \mathrm{mg} / \mathrm{mL}$ MTT was added to each well and incubated for $10 \mathrm{mins}$ at $37{ }^{\circ} \mathrm{C}$. After discarding the medium, a volume of $150 \mu \mathrm{L}$ DMSO was added to each well. The plates were placed on a shaker to mix 10 mins before the absorbance was 
measured at $570 \mathrm{~nm}$ and $650 \mathrm{~nm}$ in a microplate reader (Tecan Group Ltd.) to detect the content of formazan generated in each well. The data expressed as mean \pm SEM were obtained from three independent culture, with six replicates each. Four-parameter nonlinear regression with Prism (Version 7.03, GraphPad Software Inc., San Diego, CA) was used to fit the curves.

\section{Data Analysis}

For HEK 293 cell lines results, the curves of initial rate and amplitude as function of test concentration were fitted by nonlinear regression with four-parameter equation using Prism GraphPad, unpaired t-test assuming a Gaussian distribution were used to compare the rates and amplitudes between TBP and TBP plus caffeine treatments.

The SCO data from primary cultured cortical neurons were fitted by nonlinear curve fitting using Graph Pad Prism software (Version 7.03; GraphPad Software Inc., San Diego, CA). Potency/efficacy values were obtained by the nonlinear regression with three-parameter or four-parameter equation as described below. One-way ANOVA followed by post hoc Bonferroni's multiple comparisons test was used to compare differences between compounds $(\mathbf{3}, \mathbf{4}$ and $\mathbf{5})$ and Veh (0.1\% DMSO).

Potency values (Fig 3, 4, 6 and 7) were determined by nonlinear regression with four-parameter equation using Prism Graph Pad 7.03;

$$
y=\frac{\mathrm{A}_{1}-\mathrm{A}_{2}}{\left(1+10^{\log \mathrm{EC}_{50}-\mathrm{X}}\right) * \text { HillSlope }}+\mathrm{A}_{2}
$$

or

$$
y=\frac{\mathrm{A}_{1}-\mathrm{A}_{2}}{\left(1+10^{\log \mathrm{C}_{50}-\mathrm{X}}\right) * \text { HillSlope }}+\mathrm{A}_{2}
$$


where y was the response that decreased with the log concentration of compound $\mathbf{1}$ and 2 in Figure 6 and Figure $\mathbf{7 C}$, and that increased with the $\log$ concentration of compound 1 and $\mathbf{2}$ in Figure 7B and the log concentration of compound $\mathbf{1}$ in Figure $\mathbf{3}$ and 4, $\mathrm{A}_{1}=$ Maximum response; $\mathrm{A}_{2}=$ Minimum response; $\mathrm{EC}_{50}=$ Half-maximal effective concentration; $\mathrm{IC}_{50}=$ Half-maximal inhibitory effect concentration; HillSlope $=$ Slope factor or Hill slope (unitless).

For Figures 5 whose potency values were determined by nonlinear regression with three-parameter equation using Prism Graph Pad 7.03:

$$
y=\frac{A_{1}-A_{2}}{1+10^{\log I C_{50}-X}}+A_{2}
$$

where, $\mathrm{y}$ was the response that decreased with the log concentration of ryanodine; $\mathrm{X}=$ the $\log$ of concentration of ryanodine; $\mathrm{A}_{1}=$ Maximum response; $\mathrm{A}_{2}=$ Minimum response; $\mathrm{IC}_{50}=$ Half-maximal inhibitory concentration. 


\section{Supplementary Results}

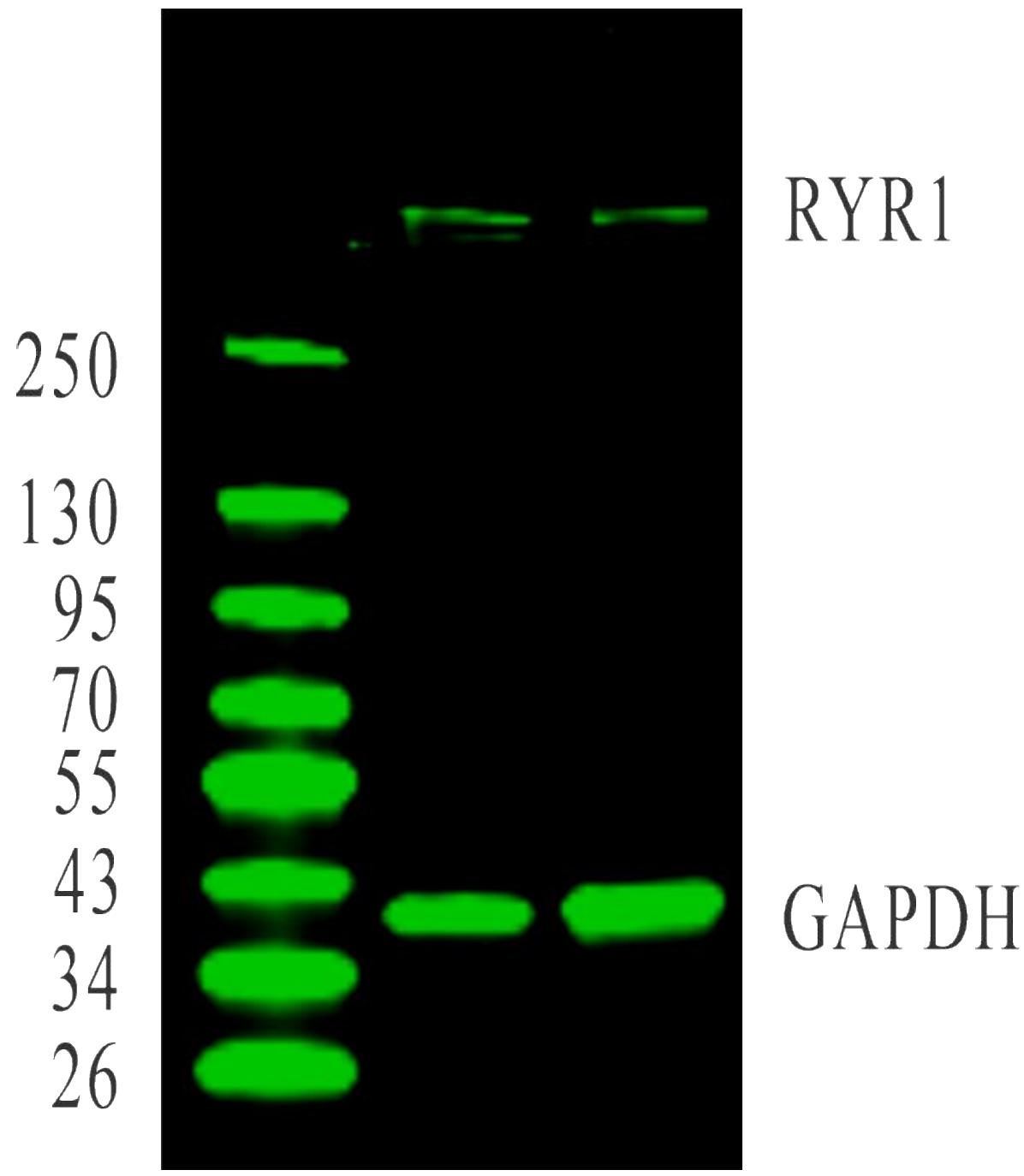

Figure S1 Representative western blot probed with RYR monoclonal antibody

34C and GAPDH polyclonal antibody showing molecular mass markers (in

kDa). The apparent mass of the RYR immunoreactive band is extrapolated to $\sim 565$

kDa, consistent with monomeric RYR1. 

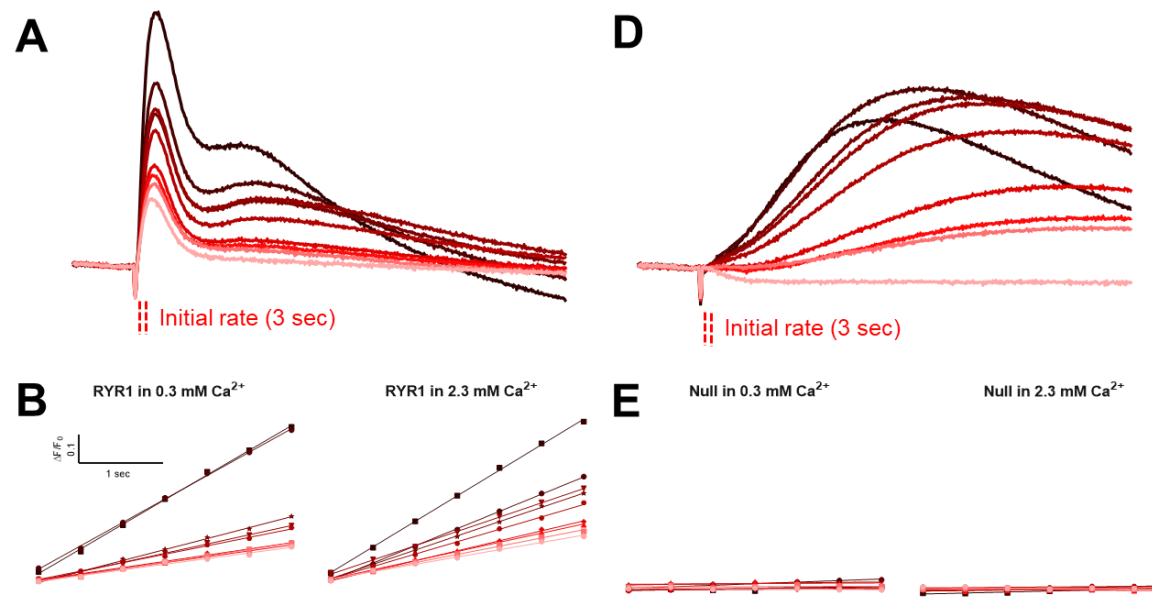

Null in $2.3 \mathrm{mM} \mathrm{Ca}^{2+}$
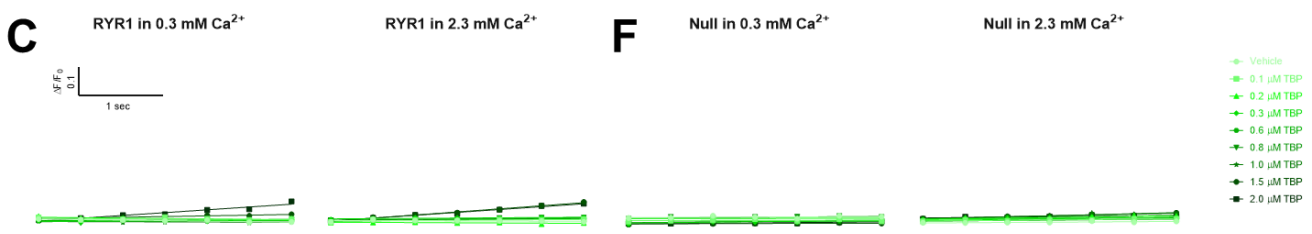

Figure S2 Initial rates of $\mathrm{Ca}^{2+}$ transients induced by TBP in null and RYR1expressing HEK293 cell lines. (A) and (D) are representative traces respectively showing $\mathrm{Ca}^{2+}$ transients induced by caffeine + Veh/TBP in RYR1-expressing and null HEK293 cell lines with $2.3 \mathrm{mM}$ external $\mathrm{Ca}^{2+}$. The initial three seconds of data after addition of caffeine + Veh/TBP were plotted in (B) and (E) for RYR1-expressing and null HEK293 cell lines, respectively. (C) and (F) are the plotting of initial three seconds of $\mathrm{Ca}^{2+}$ release triggered by Veh/TBP alone in RYR1-expressing and null HEK293 cell lines, respectively. 

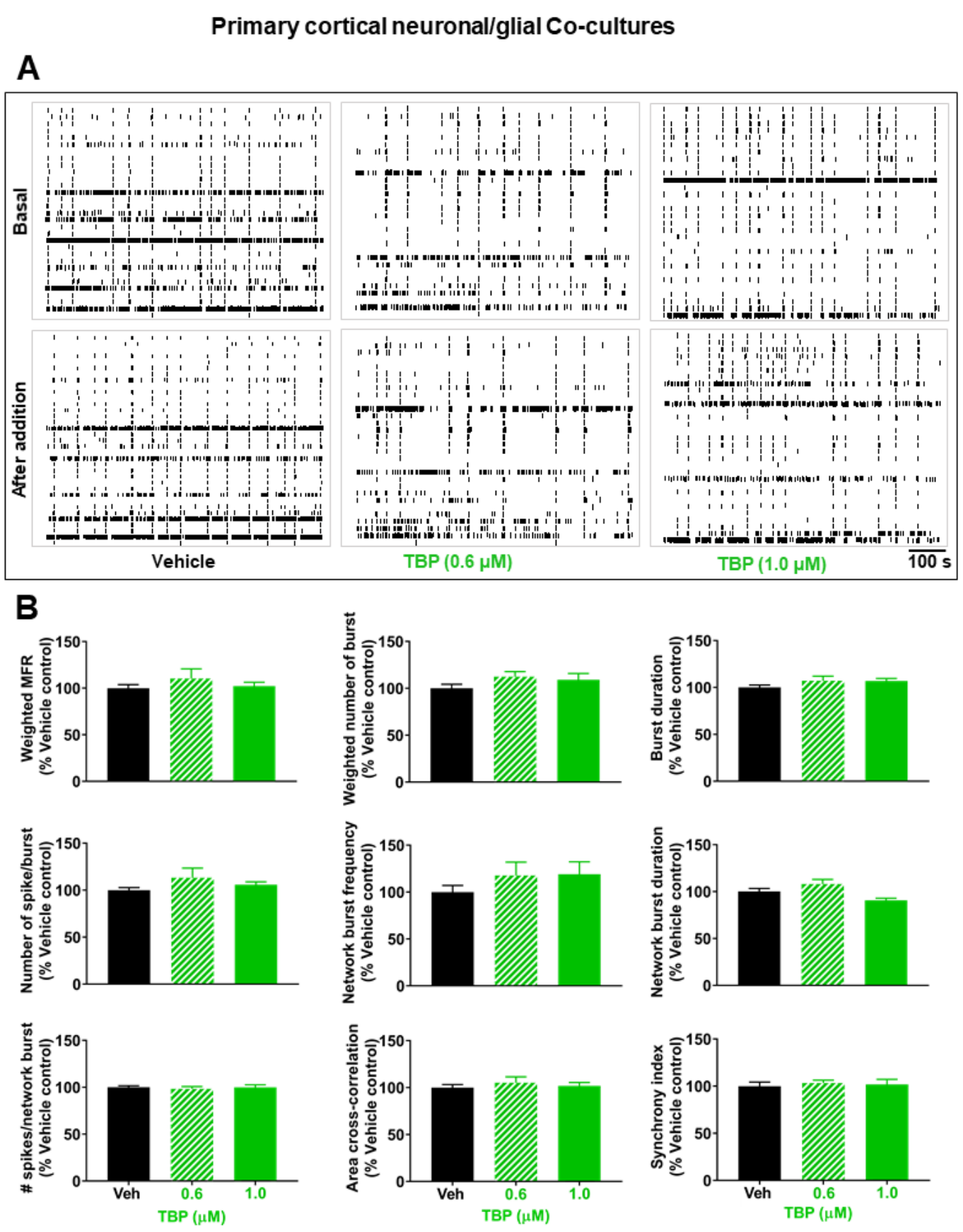

Figure S3 TBP at concentrations that fully inhibit SCO activity does not alter electrical spike activity of neuronal networks. (A) shows representative ESA raster plots of cortical neurons exposed to Veh (Black) or TBP (green). (B) displays parameters representing the general activity, bursting and network connectivity of neuronal activities. All the data expressed as mean \pm SEM were from four independent cultures, with four replicates for each culture. One-way ANOVA followed by Bonferroni's multiple comparisons test was used to determine the statistically difference 
of mean between Veh-treated and TBP treated groups with Graph Pad 7.03. Veh denotes $0.1 \%$ DMSO

\section{References}

1. Pessah, I. N.; Lehmler, H. J.; Robertson, L. W.; Perez, C. F.; Cabrales, E.; Bose, D. D.; Feng, W. Enantiomeric specificity of (-)-2,2',3,3',6,6'-hexachlorobiphenyl toward ryanodine receptor types 1 and 2. Chem. Res. Toxicol. 2009, 22 (1), 201-7.

2. Feng, W.; Barrientos, G. C.; Cherednichenko, G.; Yang, T.; Padilla, I. T.; Truong, K.; Allen, P. D.; Lopez, J. R.; Pessah, I. N. Functional and biochemical properties of ryanodine receptor type 1 channels from heterozygous R163C malignant hyperthermia-susceptible mice. Mol. Pharmacol. 2011, 79 (3), 420-31.

3. Zheng, J.; Chen, J.; Zou, X.; Zhao, F.; Guo, M.; Wang, H.; Zhang, T.; Zhang, C.; Feng, W.; Pessah, I. N.; Cao, Z. Saikosaponin d causes apoptotic death of cultured neocortical neurons by increasing membrane permeability and elevating intracellular $\mathrm{Ca}^{2+}$ concentration. NeuroToxicology 2019, 70, 112-121.

4. Cao, Z.; Xu, J.; Hulsizer, S.; Cui, Y.; Dong, Y.; Pessah, I. N. Influence of tetramethylenedisulfotetramine on synchronous calcium oscillations at distinct developmental stages of hippocampal neuronal cultures. NeuroToxicology 2016, 58, 11-22.

5. Zheng, J.; Yu, Y.; Feng, W.; Li, J.; Liu, J.; Zhang, C.; Dong, Y.; Pessah, I. N.; Cao, Z. Influence of nanomolar deltamethrin on the hallmarks of primary cultured cortical neuronal network and the role of ryanodine receptors. Environ. Health Perspect. 2019, $127(6), 67003$. 